Results 235 patients recruited: mean age 71.6 (SD 10.3); 61\% male; mean MMRC dyspnea scale 3.68 (SD 1.040); mean CAT score 23.49 (SD7.5). Anxiety and depression scores (HADS) were higher than population norms. Relationships between anxiety and depression, service use, demographic and health variables will be reported quantitatively and qualitatively. Conclusion(s) This analysis will provide new evidence on psychological health and service use in patients with advanced COPD. As part of an ongoing longitudinal study this data will inform trajectories of change in patient psychological health and service use to enable responsive service planning.

\title{
P 024 DEPRESSION AND ANXIETY: IMPACT ON SERVICE USE IN PATIENTS WITH ADVANCED CHRONIC OBSTRUCTIVE PULMONARY DISEASE
}

Carole Gardener, ${ }^{1}$ Morag Farquhar ${ }^{1}$ Hanne Holt Butcher ${ }^{1}$ Caroline Moore ${ }^{1}$ Gail Ewing, ${ }^{2}$ Patrick White, ${ }^{3}$ Sophie Howson, ${ }^{4}$ Ravi Mahadeva, ${ }^{5}$ Sara Booth, ${ }^{5}$ Peter Burge, ${ }^{6}$ Silvia Mendonca, ${ }^{1}$ on behalf of the Living with Breathlessness study team. ${ }^{1}$ Primary Care Unit, University of Cambridge; ${ }^{2}$ Centre for Family Research, University of Cambridge; ${ }^{3}$ King's College London; ${ }^{4}$ Cambridgeshire Community Services; ${ }^{5}$ Cambridge University Hospitals NHS Foundation Trust; ${ }^{6}$ RAND Europe

10.1136/bmjspcare-2014-000838.27

Introduction There is a high prevalence of anxiety and depression among people with Chronic Obstructive Pulmonary Disease (COPD). Anxiety and depression are associated with negative health outcomes and increased service use, particularly hospital admissions. Other demographic and health variables are also significant in this relationship. However, less is known about the nature of these relationships when COPD is advanced.

Aim: To identify the relationship between anxiety and depression and service use in patients with advanced COPD, and the role of demographic and health variables.

Methods: A population-based cohort of patients with advanced COPD recruited to the mixed-method Living with Breathlessness Study completed the Hospital Anxiety and Depression Scale (HADS) and reported hospital and community service use and experiences. Patient demographics and health variables were also collected. Quantitative analyses will explore relationships between anxiety and depression, service use, and demographic and health variables. Purposively sampled qualitative data analysed using a framework approach. 